\title{
¿Códigos en la Posmodernidad? Opiniones de Psicólogos/as Acerca del Código de Ética Profesional del Colegio de Psicólogos de Chile (A.G.) $)^{1,2}$
}

\author{
Post-Modern Codes? Psychologist's Opinions About the Chilean Psychologist's \\ Association Ethics Code
}

\author{
Katherine Alvear P. \\ Universidad Diego Portales, Chile \\ Diana Pasmanik V., María Inés Winkler M., Bárbara Olivares E. \\ Universidad de Santiago de Chile, USACH, Chile \\ (Rec: 24 marzo 2008. Acep: 06 noviembre 2008)
}

Ni la condición posmoderna puede prescindir de la idea de consenso al elaborar su idea de justicia. (Adela Cortina, 2000).

\section{Resumen}

Se presentan los resultados de la aplicación de un cuestionario diseñado para conocer la opinión de psicólogos/as chilenos/as acerca del Código de Ética del Colegio de Psicólogos de Chile (A.G.) y su propensión a adscribir a sus normas. La muestra fue obtenida mediante un muestreo no probabilístico, por disposición de autoselección, compuesta por 170 psicólogos/as, titulados/as en más de 20 universidades distintas, principalmente sujetos jóvenes y que se desempeñan en una amplia gama de ámbitos profesionales. Los resultados muestran que los profesionales que ejercen en el ámbito clínico y afiliados al Colegio de Psicólogos, presentarían una mayor propensión a adscribir a la norma, en comparación con quienes trabajan en otros campos profesionales. Se concluye que tanto el contexto de la post modernidad como la diversificación de roles profesionales obliga a repensar la dimensión ética presente en el ejercicio de la profesión en la actualidad.

Palabras clave: código de ética, posmodernidad, profesiones, opinión

\begin{abstract}
The results of the application of a questionnaire design to access to the opinion of Chilean Psychologists about the Code of Ethics Code and their propensity to abide by it are presented. The sampling was obtained through a non random survey, by self selection, and was integrated by 170 psychologists graduated in more than 20 different universities, preferably young adults that work in a wide variety of professional fields. The results show that the professionals that work as clinical psychologists and are members of the Chilean Psychologist's Association, tend to more strongly abide by the norms of the association, in comparison with those that work in other areas of the profession. The conclusion is that both the postmodern context as well as the diversification of professional roles obligates to rethink the ethic dimension in the exercise of the profession at the present time.
\end{abstract}

Key words: Ethic code, post modernity, professional, opinion

\footnotetext{
Los resultados presentados en este artículo forman parte del Proyecto Fondo Nacional de Ciencia y Tecnología (FONDECYT) n 1050009 intitulado "La Ética en la Formación y Ejercicio Profesional de la Psicología en Chile: un Diagnóstico Multidimensional”, financiado por CONICYT (Comisión Nacional de Investigación Científica y Tecnológica, Chile) y que cuenta con el patrocinio del Colegio de Psicólogos de Chile (AG). Tal patrocinio se manifestó, en este caso, en el envío del instrumento aplicado a la base de datos del Colegio de Psicólogos, así como en apoyo administrativo para tales efectos.

2 Correspondencia: Dra. María Inés Winkler, Escuela de Psicología, Universidad de Santiago de Chile. Dirección: Av. Ecuador 3650 Estación Central, Santiago. Correo electrónico: mariaines.winkler@gmail.com.
} 
A la modernidad, período en el cual el pensamiento sitúa al sujeto y a la razón como ejes centrales de los procesos de construcción del conocimiento, subyace la idea de que existe un desarrollo y bienestar posibles implicados en el progreso de la humanidad, al cual debe necesariamente apuntar el crecimiento tecnológico y científico. En este contexto se homologa desarrollo científico con "hacer el bien", lo cual redunda en ciertas maneras de concebir el propósito de las profesiones, en tanto aplicación de los nuevos saberes descubiertos. Estas ideas se actualizan con los cambios que tensionan y hacen necesario redefinir el esquema moderno, generando un nuevo contexto: la posmodernidad. Ha sido entendida como un estilo de pensamiento, escéptico ante las nociones clásicas de verdad, razón, identidad y objetividad, de la idea de progreso universal o de emancipación y de los grandes relatos o de los sistemas definitivos de información. De este modo, la posmodernidad considera al mundo contingente, inexplicado, diverso, inestable, indeterminado; como un conjunto de culturas desunidas o de interpretaciones diversas acerca de realidades también múltiples no exentas de intereses políticos e ideológicos (Eagleton, 1997, Amorós, 1995), que ante las certezas modernas, se transmudan en incertidumbres y relativismos.

Así, el lugar de las profesiones y su aporte al progreso y bienestar humano es puesto en tela de juicio y, de igual modo, obliga a repensar la dimensión ética, pues el comportamiento ético correcto, antes único e indivisible, comienza a evaluarse como "razonable desde el punto de vista económico", "estéticamente agradable", "moralmente adecuado". Las acciones pueden ser correctas en un sentido y equivocadas en otro (Sanabria, 2001; Bauman, 2004).

Este nuevo contexto hace necesaria la problematización de lo establecido como actuar profesional idóneo, tradicionalmente plasmado en los códigos deontológicos. El estudio que reportamos en este trabajo tuvo como objetivo conocer y describir las opiniones de psicólogos/as chilenos/ as acerca del Código de Ética del Colegio de Psicólogos de Chile (A.G.), así como también la propensión a adscribir a las normas deontológicas allí expresadas.

\section{Profesiones, ética y postmodernidad}

Existe cierto consenso en la definición de profesión como una actividad socialmente institucionalizada, que proporciona de manera específica bienes y servicios necesarios a la sociedad y que requieren un largo proceso de formación y especialización acreditado (Gyarmati, 1984; Cortina, Torralba y Zugasti, 1997; Fernández Pérez, 2001; Hirsch, s/f y 2003).

Gyarmati (1984) plantea que las profesiones son esenciales para asegurar la vida y el bienestar de los miembros de la sociedad, anteponiendo los intereses de los clientes a toda otra consideración. La ética profesional entonces, es condición de posibilidad y realización del bien social y la justicia, constituyendo, asimismo, un marco reflexivo para la toma de decisiones en el contexto del ejercicio profesional (Hirsch, 2003).

En este punto, adquiere relieve establecer la diferencia entre ética profesional y deontología profesional. A la primera subyacen aspectos aspiracionales, en tanto por medio de su discurso se indica en qué consiste una buena actuación; mientras que la segunda refiere a aspectos normativos, al formular las obligaciones y deberes del/a profesional por medio de un código avalado por el gremio (García y Cerón, 2005). Hortal (2003, en García y Cerón, 2005) sostiene que para apuntar al buen ejercicio profesional es aconsejable combinar los principios éticos con las normas deontológicas $\mathrm{y}$, al mismo tiempo, inscribir las normas deontológicas en el horizonte de las aspiraciones éticas.

Según Hirsch (2003), además, es preciso que se produzca una identificación con las pautas ideales de la profesión y un alto grado de autocontrol de la conducta, mediante la interiorización de un código ético / deontológico. Ello se logra a través de un proceso de socialización en los valores de la profesión y también, aunque en menor grado, por el control externo de instituciones gremiales, científicas o académicas.

Estos planteamientos aparecen como consistentes con el pensamiento moderno, que otorga importancia a las profesiones en su calidad de oferentes de servicios, para promover el progreso tanto de la sociedad como de la ciencia, desde espacios de saber restringidos, pero especializados (Weber, 2007). En otras palabras, la ciencia de la disciplina profesional aportaría métodos para pensar, pero por sobre todo, para examinar la conciencia sobre el sentido del propio quehacer humano (Abellán en Weber, 2007).

No obstante, tales concepciones acerca de la ciencia y las profesiones y su dimensión ética se trastrocan en el mundo postmoderno. En éste importa la felicidad y, en la medida en que no se acepta un fundamento ontológico de la realidad, tampoco se acepta una ética, sino se propone una pluralidad de éticas o microéticas, según la concepción particular de cada quién. Esta concepción rechazará lo prescriptivo en privilegio de lo descriptivo, lo fácil y lo placentero (Sanabria, 2001).

Bauman (2004), en su obra Ética postmoderna, afirma que a diferencia de lo ocurrido en la modernidad, en esta época las personas ya no están dispuestas a hacer un esfuerzo por alcanzar ideales morales, ni defender por valores morales. Este período "posterior al deber" admite apenas un vestigio de moralidad, una moralidad "minimalista". A pesar de su pretensión de arrancar la máscara de la ilusión, reconociendo como falsas ciertas pretensiones y objetivos que no pueden alcanzarse, el autor considera posible hacer visibles las fuentes de fuerza moral que se encontraban ocultas en la filosofía ética moderna, de modo de abordarlas en una forma novedosa. En este sentido, las posibilidades de "moralización" de la vida social podrían eventualmente mejorarse, instalando la interrogante acerca de cómo se 
inscribirá la postmodernidad en la historia: ya sea con el estatuto de ocaso o renacimiento de la moralidad.

Por su parte, Adela Cortina (2003) declara que, frente a la posmodernidad, la ética de las profesiones requiere necesariamente inscribirse en una ética ciudadana, capaz de involucrarse en las tareas públicas de la sociedad en su conjunto, mediante el accionar de todas sus tramas asociativas, en las que es preciso articular las instancias gremiales y a los actores y actoras sociales involucrados, en este caso psicólogos/as y usuarios/as de sus servicios.

\section{Código de Ética del Colegio de Psicólogos de Chile (A.G.)}

Desde sus inicios, el Colegio de la Orden incorporó la preocupación ética, en forma explícita, en varios artículos de la Ley 17.033 que creó el Colegio de Psicólogos. Asimismo, la revisión de actas y documentos dan cuenta de los esfuerzos por generar normas y principios aspiracionales orientados a forjar un ejercicio profesional óptimo, desde los comienzos de la existencia del colegio (Winkler y Castillo, en preparación). La primera Comisión de Ética, integrada por un pequeño grupo de asociados, elaboró los primeros documentos normativos. Posteriormente, a mediados de los años 70 se aprobó el primer Código de Ética Profesional del Colegio de Psicólogos de Chile (1975); a fines de 1976 se elaboró las Normas Éticas en una traducción casi textual de las normas de la APA (American Psychological Association); y en 1981 se sancionó una reformulación del Código de Ética (Acta 22 de enero 1981). Ulteriormente, en 1997 se redactó un documento más actualizado, complejo y extenso, sancionado en forma definitiva el año 1999 y que corresponde al Código de Ética Profesional vigente hasta hoy (Colegio de Psicólogos de Chile, 1999).

El código vigente en Chile enfatiza tanto aspectos deontológicos (normativos, presentes en sus Normas Éticas Específicas), como aspectos educativos (Normas Éticas Generales) y propone principios y aspiraciones (Principios Generales). Está pensado como un instrumento facilitador de un buen ejercicio y de la resolución de dilemas éticos en el quehacer profesional.

A diferencia de otros países, en Chile no existen lineamientos $\mathrm{u}$ orientaciones para el quehacer ético en áreas específicas, como por ejemplo, las orientaciones éticas especiales para psicólogos/as forenses creadas por Committee on Ethical Guidelines of Division 41 y la American Academy of Forensic-Psychology (1991); o como las guías éticas para psicólogos que proveen servicios a través de medios electrónicos (2006) o las guías para la práctica psicológica ética con mujeres, ambas de la Canadian Psychological Association ( CPA, 2007).

\footnotetext{
Para profundizar en estos y otros códigos por áreas específicas, sugerimos consultar la página http://www.kspope.com/ethcodes/index.php de Kenneth Pope.
}

Por otra parte, en psicología, es recurrente la revisión y actualización de los códigos de ética por equipos de expertos/as, utilizando como estrategia la comparación de distintos códigos de conducta, y siguiendo, con mucha frecuencia, el modelo de la APA (Leach y Harbin, 1997; Pettifor, 2004).

Algunos ejemplos de estas actualizaciones son aquellas llevadas a cabo por las Asociaciones de Psicólogos Canadiense, Americana, Neozelandesa y Británica. Así, el Código de Ética de la Asociación Psicológica Canadiense (CPA) fue modificado en el año 2000; en el año 2002 se revisó los códigos de la Asociación Psicológica Americana en Estados Unidos (APA) y el código de la Sociedad Psicológica de Nueva Zelanda (New Zealand Psychological Society); mientras que en el año 2006 se efectuó una modificación del Código Ético de la Sociedad Psicológica Británica (British Psychological Society, 2006).

Por su parte, la Sociedad Mexicana de Psicología (2007), a partir de un estudio empírico, además de evaluar el Código de Ética del Psicólogo, identificó ámbitos nuevos sobre los que era necesario establecer normas de conducta. Para ello se basaron en principios propuestos por Sinclair y Pettifor (1991) y revisaron el código ético, formulándose las siguientes interrogantes: ¿educa el código a los usuarios potenciales?, ¿se vinculan las normas explícitamente con los principios generales?, ¿se enuncian claramente las normas?, ¿enuncia el código valores morales?, ¿apoya el código a la mayoría de las aéreas de la psicología?, y, finalmente, ¿el código se basa en casos reales que reflejen la práctica profesional actual?

Se concluye en dicho estudio que un nuevo código debe necesariamente tomar en cuenta aspectos que el anterior ignoró, así como también, incluir áreas de aplicación más allá de la terapia individual, tales como la industrialorganizacional, educativa, comunitaria, de la salud, etc. (Sociedad Mexicana de Psicología, 2007)

Por otra parte, un área especialmente fructífera en la reflexión e investigación acerca de los códigos de conducta ha sido la empresarial y de los negocios, que ha contribuido en la descripción y desarrollo de métodos para generar códigos (Rodríguez y Díaz, 2004), en el cuestionamiento acerca de su efectividad (Kaptein y Schwartz, 2008) y de su valor como garantes de comportamientos éticos consistentes en los empleados (Farrell, Cobbin y Farrell, 2002) y sobre las actitudes hacia los códigos éticos (Peppas, 2003).

En Chile no se han efectuado modificaciones al código desde el año 1999. Sin embargo, nos parece interesante destacar algunos resultados de un estudio de tipo exploratorio, desarrollado en el marco del Primer Encuentro Nacional de Psicología Jurídica, organizado el año 2004 por la Asociación Chilena de Psicología Jurídica, que pretende aportar a una revisión preliminar del código de ética, que considere las transformaciones psicosociales, culturales y económicas experimentadas en los últimos años en nuestro 
país. Se buscó conocer el nivel de información que poseen algunos psicólogos/as y estudiantes chilenos/as respecto de determinados aspectos éticos involucrados en el quehacer del profesional en el área jurídica (Fassler y Rivas, 2005). Los resultados indican que la mayoría de los/as encuestados/ as asevera conocer el Código de Ética y haber leído todos los artículos; sin embargo, existe un porcentaje importante de personas que no. Además, respecto de la función orientadora del código, un $82.4 \%$ responde que éste no sería útil para el enfrentamiento y resolución de problemas y dilemas éticos relacionados con el ejercicio jurídico y forense.

Lo anterior evidencia la necesidad de generar un cuerpo de datos empíricos respecto a la construcción y evaluación de los códigos de ética. No se dispone de mayores antecedentes, distintos de los reseñados, acerca de investigaciones previas respecto de las opiniones, actitudes, evaluaciones u otros, de psicólogos sobre los códigos de ética de Chile o de otros países. Schwartz (2004), en el ámbito empresarial, critica los estudios acerca de los códigos éticos por su énfasis en los contenidos, en lugar de acentuar las recomendaciones o efectividad percibida por sus usuarios/ as, señalando la importancia de considerar su percepción en las evaluaciones acerca de la efectividad potencial de los códigos. Concordando con ese autor, en este trabajo se supone que es posible acceder a posiciones valorativas acerca de los códigos deontológicos y que su medición puede ser relevante en la toma de decisiones para actualizar los Códigos de Ética.

Con este objetivo se diseñó un instrumento para medir las opiniones y disposición hacia los códigos de conducta de psicólogos/as chilenos/as ${ }^{4}$. Se trata de una encuesta de opinión que contiene dos dimensiones: Opiniones acerca del Código de Ética profesional y Propensión a adscribir a la norma.

El constructo de "opinión", según la RAE (Real Academia Española, 2007), se define como "dictamen o juicio que se forma de algo cuestionable", y "opinión pública", como "sentir o estimación en que coincide la generalidad de las personas acerca de asuntos determinados", donde en este caso, "la generalidad de las personas" serían los/as psicólogos/as.

La propensión a adscribir a la norma también se deja definir recurriendo a la RAE (2001), pues propender es "dicho de una persona: inclinarse por naturaleza, por afición o por otro motivo, hacia algo en particular". Así, la propensión, en este caso, se referirá a la inclinación a usar el Código de Ética como guía para la conducta profesional. Así, nuestras hipótesis sostienen que la exploración de las opiniones sobre el Código de Ética se enriquece en la medida que se da por hecho que refleja una intención o propensión a

\footnotetext{
4 El procedimiento de construcción y validación preliminar del cuestionario es documentado en el artículo Encuesta de Opinión sobre el Código de Ética Profesional del Colegio de Psicólogos de Chile (AG); de las autoras Winkler, Pasmanik, Alvear y olivares (en revisión).
}

su uso. En esta investigación se explora si la propensión a adscribir a la norma es menor cuanto más jóvenes sean los/ as psicólogos/as, asumiendo que es más factible que hayan sido socializados en un contexto posmoderno.

También hipotetizamos que existiría una valoración más positiva del Código de Ética del Colegio de Psicólogos de Chile por parte de los psicólogos/as especializados en el campo clínico y por quienes están colegiados. Esta hipótesis posee dos fundamentos. En primer lugar, las normas específicas del código se refieren a las instancias de relación de ayuda preponderantemente en el trabajo clínico. En segundo lugar, dado el carácter voluntario de afiliación gremial, sería esperable encontrar en los colegiados una valoración más favorable del Código de Ética de la orden.

En adición a las hipótesis planteadas, surge la inquietud con respecto a la propensión de los psicólogos/as a ceñir su comportamiento a los lineamientos y normas que el Código de Ética proporciona, más allá de su opinión acerca del Código en sí. Esto a partir de la relatividad de los valores que caracteriza el marco de la posmodernidad donde el desarrollo de la técnica, la diseminación de la informática y las movilidades económico-sociales han convertido el mundo de los valores en un terreno de permanente inestabilidad (Díaz, 1999).

\section{Método}

Se trata de un estudio descriptivo, con un diseño transeccional correlacional (Hernández, Fernández y Baptista, 1991) que, a través de la aplicación de un cuestionario, mide las opiniones de los profesionales psicólogos acerca del Código de Ética Profesional chileno y su propensión a adscribir a sus normas.

\section{Instrumento}

A partir de insumos obtenidos en un estudio ejecutado por el mismo equipo investigador durante los años 2003 y 20045, se elaboró una encuesta de opinión que presenta dos dimensiones: Propensión a Adscribir a la Norma y Opiniones acerca del Código de Ética.

El instrumento contiene 12 reactivos en escala tipo Likert, donde "1" equivale a "Totalmente en Desacuerdo" y "5" a "Totalmente de Acuerdo"; y al mismo tiempo, mediante preguntas dicotómicas, explora la pertenencia y participación gremial de los y las encuestadas; y contiene una pregunta abierta acerca de los Principios que se recuerda del código deontológico. El cuestionario se presenta mediante una hoja de consentimiento informado, seguida de un protocolo que permite caracterizar a los y las profesionales según edad, sexo, universidad de procedencia, año de titulación y área de desempeño (ver anexo).

\footnotetext{
5 Proyecto FONDECYT 1030658: "Ética y Género en Psicología: Historia y Representaciones Actuales".
} 
Sus propiedades psicométricas tienen una validez factorial de $55.0 \%$ y una consistencia interna 0.8 según el coeficiente alpha de Cronbach.

\section{Participantes}

La muestra fue obtenida mediante un muestreo no probabilístico, por disposición, de autoselección (Ruiz y Morillo, 2004). Se trata de 170 sujetos, 70.6\% mujeres y $28.8 \%$ hombres $(0.6 \%$ no responde al ítem); con una edad promedio de 37.4 años, con mediana y moda iguales a 33 años; donde el 55.3\% tiene una edad entre 23 y 35 años y el $44.1 \%$ posee entre 36 y 75 años.

Los psicólogos y psicólogas de la muestra se han titulado entre el año 1961 y el 2007, siendo la media el año 1995. El $50.5 \%$ de la muestra se ha titulado a partir del año 2000.

Respecto de las universidades de procedencia de estos profesionales, se obtiene que un $60 \%$ de los/as encuestados/ as proviene de 20 instituciones privadas, un $36.5 \%$ de ocho universidades públicas y un $2.9 \%$ de cuatro universidades extranjeras. Un $33.5 \%$ proviene de las 2 universidades existentes hasta el año 1981, tal como se desprende de la Tabla 1.

Tabla 1. Universidades de Procedencia de los encuestados

\begin{tabular}{|c|c|c|}
\hline Universidad de Procedencia & Frecuencia & $\%$ \\
\hline U. de Chile & 29 & 17.1 \\
\hline Pontificia U. Católica de Chile & 28 & 16.5 \\
\hline U. Diego Portales & 13 & 7.6 \\
\hline U. de Santiago de Chile & 9 & 5.3 \\
\hline U. de La Frontera & 9 & 5.3 \\
\hline U. Nacional Andrés Bello & 9 & 5.3 \\
\hline U. Central & 8 & 4.7 \\
\hline U. de Tarapacá & 7 & 4.1 \\
\hline U. de Concepción & 7 & 4.1 \\
\hline U. de Las Américas & 6 & 3.5 \\
\hline U. Santo Tomás & 5 & 2.9 \\
\hline U. Extranjeras & 5 & 2.9 \\
\hline U. de Viña del Mar & 4 & 2.4 \\
\hline U. del Desarrollo & 4 & 2.4 \\
\hline U. Gabriela Mistral & 3 & 1.8 \\
\hline U. La República & 3 & 1.8 \\
\hline U. Católica de Valparaíso & 3 & 1.8 \\
\hline U. de La Serena & 2 & 1.2 \\
\hline U. Bolivariana & 2 & 1.2 \\
\hline U. San Sebastián & 2 & 1.2 \\
\hline U. de Valparaíso & 2 & 1.2 \\
\hline UNIACC & 2 & 1.2 \\
\hline Otras universidades & 7 & 4.1 \\
\hline No responde & 1 & 0.6 \\
\hline Total & 170 & 100 \\
\hline
\end{tabular}

Nota: en la categoría “Otras universidades" se agrupó a aquellas cuyos porcentajes eran iguales a 0.6

Por otra parte, respecto de las áreas de desempeño, la mayoría, es decir, el 54.1\% $(\mathrm{n}=92)$ menciona sólo una y un $41.2 \%(\mathrm{n}=70)$ menciona, al menos, dos áreas de ejercicio profesional, que suman 153 alusiones a áreas de desempeño. En la Tabla 2 se expone la información obtenida. 
Tabla 2. Áreas de desempeño de los encuestados.

\begin{tabular}{lcc}
\hline & $\begin{array}{c}\text { Sólo } \begin{array}{c}\text { área de desempe- } \\
\text { ño }(\mathrm{n}=92)\end{array} \\
\text { 2 o más áreas de desempeño (n=70) }\end{array}$ \\
\hline Psicología clínica & 45.7 & 38.6 \\
Psicología laboral y de las organizaciones & 17.4 & 12.4 \\
Psicología forense, criminológica y jurídica & 12.0 & 9.1 \\
Educacional & 9.8 & 9.1 \\
Docencia, investigación & 7.5 & 19.0 \\
Psicología social & 2.2 & 0.7 \\
Salud & 2.2 & 3.3 \\
Psicología del deporte & 1.1 & \\
Administración & 1.1 & 3.3 \\
Psicología comunitaria & 1.1 & 1.3 \\
Mediación familiar & & 1.3 \\
Género & & 0.7 \\
Infancia & & 0.7 \\
Administración & & 0.7 \\
Emergencia & 100 & 100 \\
Total & & \\
\hline Nota: Resultados en porcentajes. El porcentaje de la columna “2 o más áreas de desempeño" se estimó a partir del número de alusiones &
\end{tabular}

El $81.8 \%$ de los/as encuestados/as dice conocer el Código de Ética Profesional, mientras que el $67.1 \%$ de la muestra está afiliado al Colegio de Psicólogos (A.G.).

\section{Procedimiento:}

Se utilizó dos estrategias para la aplicación de los cuestionarios. La primera de ellas fue vía correo electrónico. Con este propósito, se recurrió en dos ocasiones a la base de datos de correos electrónicos de los/as profesionales colegiados/as $(\mathrm{N}=2.375)$, facilitada por el Colegio de Psicólogos de Chile (A. G.) y a la dirección de email de la bolsa de trabajo de la página de comunitarios.cl, que entonces contaba con 2.000 suscritos. De manera paralela se envió a la lista de correos del Journal Psicológico ${ }^{6}(\mathrm{~N}$ $=361$ ). Las dos últimas bases contienen datos tanto de colegiados/as como no colegiados/as.

La administración electrónica de la encuesta se realizó entre los meses de octubre de 2006 y octubre de 2007, obteniéndose una muestra de 134 cuestionarios, indicando una tasa de respuesta aproximada del $1.8 \%$.

La segunda estrategia consistió en la aplicación de encuestas en seminarios y charlas, mediante la cual se obtuvo

\footnotetext{
6 Agradecemos especialmente al colega Marcelo Urra, quien gentilmente facilitó el acceso a la base de datos de correos electrónicos perteneciente al Journal Psicológico (www.psicologico.cl).
}

un total de 36 cuestionarios adicionales, configurando el total de encuestas $(\mathrm{N}=170)$.

\section{Análisis de los Datos}

Se utilizó el paquete estadístico para ciencias sociales (SPSS, v. 13.0). Se estimó el estadístico $X^{2}$ para analizar las características de la muestra. Respecto de los itemes, se calculó frecuencias y medidas de tendencia central así como también se realizó la prueba $t$ para muestras independientes, a fin de determinar si existían diferencias significativas en relación a los reactivos, por las variables sexo, edad, área de desempeño y afiliación gremial de los y las encuestadas.

\section{Resultados}

En lo siguiente, los resultados son presentados en tres partes. La primera de ellas describe la pertenencia y participación de los/as encuestados en lo referido al gremio 1 y el conocimiento del Código de Ética Profesional. En una segunda parte, proporcionamos los resultados de la encuesta propiamente tal para la muestra total; y en una tercera y última parte, según diferentes segmentaciones de la muestra: edad y sexo; afiliación al Colegio de Psicólogos y área de desempeño. 


\section{Primera parte: en relación al Código de Ética}

Profesional y al gremio

El $67.1 \%$ de la muestra está afiliado/a al Colegio de Psicólogos (A.G.), de estos 114 sujetos, 96 (84.2\%) indican

año de colegiatura; información que se distribuye tal como se expone en la Figura 1.

Figura 1. Gráfico Año de colegiatura

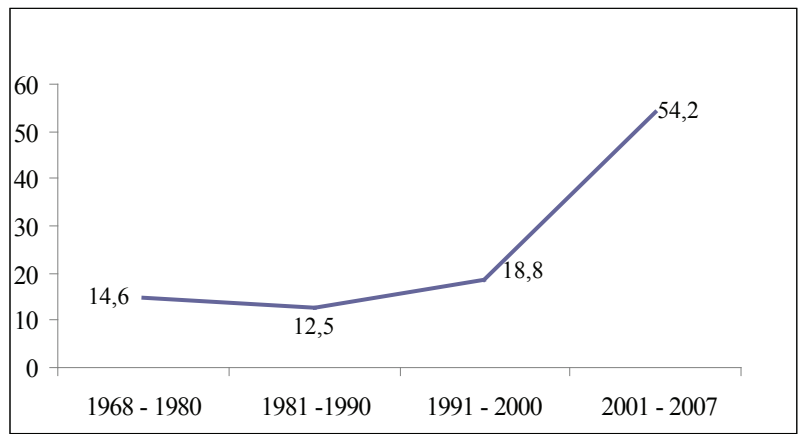

Nota: Resultados en Porcentajes.

Psicólogos/as afiliados/as al Colegio de Psicólogos de Chile ( $\mathrm{n}=96)$

Encontramos diferencias significativas por rango etario, pues hay una mayor proporción de colegiados/as entre personas mayores de 36 años, respecto de aquellas menores de
35 años, tal como se expresa en la Tabla 3 y según evidencia el estadístico $X^{2}(1, \mathrm{~N}=168) 23.17, \mathrm{p}<.01$,

Tabla 3. Afiliación al Colegio de Psicólogos de Chile según edad de los/as encuestados/as

\begin{tabular}{lccc}
\hline & Edad $=35$ años & Edad $=36$ años & Total \\
\hline Colegiado/a & 48 & 65 & 113 \\
No Colegiado/a & 45 & 10 & 55 \\
Total & 93 & 75 & 168 \\
\hline
\end{tabular}

Respecto de la participación de los/as colegiados/as, un $14.9 \%$ reporta que asiste a las actividades organizadas por el Colegio de Psicólogos, el 56\% dice pagar las cuotas, el $86.8 \%$ que visita la página web y el $90.4 \%$ que lee los boletines emitidos por la asociación gremial.
El $81.8 \%$ de los y las psicólogas responde que conoce el Código de Ética Profesional; un $76.5 \%$ señala que lo ha leído completo, al menos una vez, y un $62.4 \%$ reporta poseer un ejemplar o copia del código. La Tabla 4 contiene esta información, donde es posible apreciar diferencias significativas entre los psicólogos colegiados y no colegiados.

Tabla 4. Conocimiento acerca del Código de Ética Profesional

\begin{tabular}{|c|c|c|c|c|c|c|}
\hline & $\begin{array}{c}\text { Total } \\
(\mathrm{N}=170)\end{array}$ & $\begin{array}{l}\text { Colegiados } \\
(\mathrm{n}=114)\end{array}$ & $\begin{array}{l}\text { No Colegiados } \\
(\mathrm{n}=55) \# \text { Una } \\
\text { persona no ex- } \\
\text { plicita si está o no } \\
\text { afiliada al Colegio } \\
\text { de Psicólogos de } \\
\text { Chile (A.G.).\# }\end{array}$ & $X^{2}$ & $g l$ & $p$ \\
\hline Conoce Código de Ética Profesional & 81.8 & 86.0 & 72.7 & Ns & 2 & 0.61 \\
\hline Posee un ejemplar o copia & 62.4 & 71.7 & 43.6 & $13.61 * *$ & 2 & 0.001 \\
\hline Ha leído el Código completo al menos una vez & 76.5 & 82.5 & 63.6 & $7.93 * *$ & 2 & 0.01 \\
\hline
\end{tabular}

Nota: Resultados en porcentajes. $* * \mathrm{p}<.01$, dos colas.

Del total de la muestra, un $29.4 \%$ no responde o no recuerda la última vez que consultó el Código de Ética Profesional.
Un 44.4\% señala que lo consultó durante el último año. Más detalle de esta información se muestra en la Tabla 5. 
Tabla 5. Última vez que consultó el Código de Ética Profesional

\begin{tabular}{lccc}
\hline & $\begin{array}{c}\text { Mujeres } \\
(\mathrm{n}=120)\end{array}$ & $\begin{array}{c}\text { Hombres } \\
(\mathrm{n}=49)\end{array}$ & $\begin{array}{c}\text { Total } \\
(\mathrm{N}=169)\end{array}$ \\
\hline Durante el último año & 43.3 & 46.9 & 44.4 \\
Hace 2 años & 8.3 & 6.1 & 7.7 \\
Hace 3 años & 2.5 & 6.1 & 3.6 \\
Hace 4 años & 5.8 & 2.0 & 4.7 \\
Hace 5 años & 3.3 & 0.0 & 2.4 \\
Hace más de 5 años & 9.2 & 4.1 & 7.7 \\
No responde - No recuerda & 34.4 & 27.3 & 29.4 \\
\hline Nota: Resultados en porcentajes. Al compararlos entre hombres y mujeres no se evidencian diferencias estadísticamente \\
significativas.
\end{tabular}

Un $69.4 \%$ de los sujetos responde a la pregunta abierta que indaga sobre el recuerdo de los Principios Generales del Código de Ética Profesional, entregando 478 respuestas? de las que sólo el $27.5 \%$ corresponde efectivamente a los principios enunciados en el Código. Un detalle se presenta en la Tabla 6. tiempo, un 51.5\% problematiza la utilidad del Código de Ética Profesional, dadas las atribuciones limitadas del Colegio de Psicólogos para velar por su cumplimiento (ítem 8).

Más de la mitad de los/as encuestados/as menciona la dedicación y responsabilidad; el manejo técnico y

Tabla 6. Recuerdo de los Principios Generales del Código de Ética Profesional

\begin{tabular}{lccc}
\hline \multicolumn{1}{c}{ Principios recordados } & $\begin{array}{c}\text { Mujeres } \\
(\mathrm{n}=330)\end{array}$ & $\begin{array}{c}\text { Hombres } \\
(\mathrm{n}=178)\end{array}$ & $\begin{array}{c}\text { Total } \\
(\mathrm{N}=478)\end{array}$ \\
\hline Respeto por los derechos y dignidad de las personas & 4.8 & 2.5 & 7.3 \\
Competencia profesional & 4.4 & 2.5 & 6.9 \\
Responsabilidad social & 2.7 & 1.3 & 5.7 \\
Compromiso profesional & 2.1 & 1.7 & 3.8 \\
Independencia & 2.1 & 1.7 & 3.8 \\
Total & 16.1 & 9.7 & 27.5 \\
\hline
\end{tabular}

Nota: Resultados en porcentajes

\section{Segunda parte: resultados de encuesta de opinión acerca del Código de Ética Profesional.}

A continuación presentamos los resultados obtenidos según las escalas que conforman el instrumento (ver Tabla 7).

Según los resultados presentados en la Tabla 7, se obtiene que el $51.0 \%$ de los sujetos sostiene que rara vez enfrenta situaciones éticamente complejas en su espacio de trabajo (ítem 1). Además, se observa que alrededor de un $60 \%$ de los y las encuestadas se manifiesta contrario/a a los códigos de ética profesional (ítem 3), anteponiendo el conocimiento de las legislaciones vigentes en el área del trabajo (reactivo 7). Al mismo

\footnotetext{
Considerando que las respuestas emitidas por los/as encuestados/as no necesariamente se ajustan textualmente a los Principios del Código de Ética, el equipo investigador hipotetiza que tales respuestas corresponderían más bien a las representaciones que tienen los/as participantes acerca de los principios éticos que debieran guiar el actuar profesional de los psicólogos/as. De este modo, para el análisis de esta pregunta se combinó metodología cualitativa y cuantitativa desde la Teoría de Núcleo Central de las Representaciones Sociales. Los resultados del análisis cualitativo serán presentados en otra publicación.
}

la experiencia personal como recursos suficientes para enfrentar situaciones de cierta complejidad ética (itemes $4,5,6)$. No obstante lo anterior, como se observa en la Tabla 8 , no existe acuerdo respecto de si su propio criterio es suficiente para resolver conflictos éticos (ítem 2: 34.4\% en contra y $42.0 \%$ a favor).

En relación a las Opiniones acerca del Código de Ética, cuyos resultados son presentados en la Tabla $\mathrm{N}^{\circ} 9$, se evidencia que el $66.9 \%$ refiere que éste puede ser una herramienta útil (reactivo 9). Sin embargo, la muestra se divide en aquellos que dudan en recomendar la revisión del Código a un colega que enfrenta una situación éticamente compleja y aquellos que efectivamente lo harían ( $40.8 \%$ y $42.0 \%$, respectivamente para Neutro y A favor en reactivo 12).

La mitad de los/as encuestados/as (50.3\%) no se pronuncia acerca de si el código aborda sus necesidades desde el punto de vista ético. No existe acuerdo en relación a la claridad de los planteamientos del código, pues un $35.5 \%$ se manifiesta en una posición neutra y un $38.5 \%$ en una posición contraria a la afirmación contenida en el ítem 11. 
Tabla 7. Resumen de Resultados Escala Propensión a Adscribir a la Norma: Frecuencias y Medidas de Tendencia Central

\begin{tabular}{|c|c|c|c|c|c|c|c|}
\hline Ítem & $\begin{array}{c}\% \text { de } \\
\text { Respuestas }\end{array}$ & $\begin{array}{l}\text { Medidas } \\
\text { de } \\
\text { Tendencia } \\
\text { Central }\end{array}$ & & & & & \\
\hline & NR & En Contra & Neutro & A Favor & Media & Moda & D. E. \\
\hline $\begin{array}{l}\text { 1. En mi trabajo, rara vez enfrento situaciones } \\
\text { éticamente complejas. }\end{array}$ & 0.6 & 32.5 & 16.0 & 51.0 & 3.3 & 4 & 1.3 \\
\hline $\begin{array}{l}\text { 2. Mi propio criterio es suficiente para enfrentar } \\
\text { los problemas éticos de mi actuar profesional. }\end{array}$ & 2.4 & 34.3 & 21.3 & 42.0 & 3.1 & 4 & 1.2 \\
\hline $\begin{array}{l}\text { 3. Por regla general, soy contrario(a) a los códigos } \\
\text { de ética profesional. }\end{array}$ & 2.4 & 33.1 & 4.1 & 60.4 & 3.4 & 5 & 1.7 \\
\hline $\begin{array}{l}\text { 4. Considero que un(a) psicólogo(a) dedicado(a) } \\
\text { y responsable no necesita recurrir al código de } \\
\text { ética. }\end{array}$ & 0.0 & 33.1 & 10.7 & 56.2 & 3.4 & 4 & 1.4 \\
\hline $\begin{array}{l}\text { 5. Con un buen manejo técnico basta para cubrir } \\
\text { todos los requerimientos éticos que entraña el } \\
\text { ejercicio profesional de la Psicología. }\end{array}$ & 2.4 & 27.2 & 15.4 & 55.0 & 3.4 & 4 & 1.4 \\
\hline $\begin{array}{l}\text { 6. La experiencia personal es lo único que me per- } \\
\text { mite discernir apropiadamente frente a problemas } \\
\text { éticos en el trabajo }\end{array}$ & 1.2 & 30.8 & 14.8 & 53.3 & 3.3 & 4 & 1.3 \\
\hline $\begin{array}{l}\text { 7. Los códigos de ética profesional están demás, } \\
\text { lo que hace falta es conocer la legislación vigente } \\
\text { en el área de trabajo de cada uno. }\end{array}$ & 1.2 & 30.8 & 8.9 & 59.2 & 3.3 & 4 & 1.5 \\
\hline $\begin{array}{l}\text { 8. No tiene sentido poseer un Código de Ética, } \\
\text { cuando tenemos un Colegio de Psicólogos que } \\
\text { tiene atribuciones limitadas y sólo para sancionar } \\
\text { a sus colegiados. }\end{array}$ & 1.2 & 31.4 & 16.0 & 51.5 & 3.3 & 4 & 1.4 \\
\hline
\end{tabular}

Nota: para facilitar la lectura de los resultados, se reorganizó las respuestas de la escala Likert de las categorías "Muy en desacuerdo" y "En desacuerdo" en la columna En Contra; "Ni de acuerdo ni en desacuerdo" en términos de Neutro y "De acuerdo" y Muy de Acuerdo" como A Favor.

En líneas generales, las Opiniones acerca del Código de Ética Profesional valoran la utilidad del código deontológico del gremio; sin embargo, llama la atención que no exista un acentuado pronunciamiento valorativo acerca de las temáticas y necesidades que éste aborda.

\section{Tercera parte: resultados según segmentación de la muestra Según edad y sexo}

Se trata de una muestra joven, donde destaca que las mujeres, en compararción con los hombres, desarrollan su quehacer profesional en mayor medida en el ámbito clínico; diferencia significativa que se expresa en $X^{2}(1, \mathrm{~N}=164)$ $=4.46, \mathrm{p}<.05$.

$\mathrm{Al}$ revisar los resultados por subgrupos, de acuerdo a la prueba $t$ para muestras independientes, aparecen diferencias significativas en los resultados por la variable sexo, pues según los resultados obtenidos para el ítem 8 los hombres problematizan la utilidad del código mucho más que las mujeres, en relación a las atribuciones del Colegio de
Psicólogos para resguardar el comportamiento ético de los/ as psicólogos/as (ver Tabla 9).

Por otra parte, al dividir la muestra en dos grupos etarios, se observa que la media de respuestas para el ítem 12 es significativamente mayor para el grupo de edades sobre 36 años $(3,7)$, respecto de aquellos cuyas edades son iguales o menores a 35 años $(3,3)$. Así, el primer grupo sería más proclive a recomendar el uso del código ético como recurso para la solución de problemas éticos, manifestando una opinión más favorable.

\section{Resultados según Afiliación al Colegio de Psicólogos de Chile}

En los resultados de la prueba $X^{2}$ no se observa diferencias significativas entre los y las profesionales afiliados/ as y los no afiliados al Colegio de Psicólogos de Chile, en relación a la última vez que consultaron el Código de Ética Profesional (Tabla 11). 
Tabla 8. Resumen de Resultados Escala Opiniones acerca del Código de Ética: Frecuencias y Medidas de Tendencia Central

\begin{tabular}{|c|c|c|c|c|c|c|c|}
\hline Ítem & $\begin{array}{c}\% \text { de } \\
\text { respuestas }\end{array}$ & $\begin{array}{l}\text { Medidas } \\
\text { de } \\
\text { Tendencia } \\
\text { Central } \\
\end{array}$ & & & & & \\
\hline & NR & En Contra & Neutro & A Favor & Media & Moda & D. E \\
\hline $\begin{array}{l}\text { 9. El Código de Ética Profesional del Colegio } \\
\text { de Psicólogos es una herramienta útil que } \\
\text { orienta frente a situaciones de dilema o } \\
\text { conflicto ético. }\end{array}$ & 4.7 & 10.7 & 17.8 & 66.9 & 3.7 & 4 & 1.3 \\
\hline $\begin{array}{l}\text { 10. El Código de Ética Profesional del } \\
\text { Colegio de Psicólogos aborda todas las } \\
\text { necesidades que tenemos en materias de ética } \\
\text { profesional. }\end{array}$ & 7.1 & 30.0 & 50.3 & 13.0 & 2.6 & 3 & 1.1 \\
\hline $\begin{array}{l}\text { 11. El Código de Ética Profesional del } \\
\text { Colegio de Psicólogos es claro. }\end{array}$ & 3.0 & 38.5 & 35.5 & 23.1 & 2.7 & 3 & 1.2 \\
\hline $\begin{array}{l}\text { 12. Si viera a un(a) colega enfrentando } \\
\text { una situación éticamente compleja, le } \\
\text { recomendaría que recurriera al Código de } \\
\text { Ética Profesional del Colegio de Psicólogos. }\end{array}$ & 6.5 & 10.7 & 40.8 & 42.0 & 3.2 & 4 & 1.1 \\
\hline
\end{tabular}

Tabla 9. Psicólogos y Psicólogas: Reactivo que expresa diferencias estadísticamente significativas

\begin{tabular}{|c|c|c|c|c|c|}
\hline & & Media & $T$ & gl & Sig \\
\hline \multirow{2}{*}{$\begin{array}{l}\text { 8. No tiene sentido poseer un Código de Ética, } \\
\text { cuando tenemos un Colegio de Psicólogos que tiene } \\
\text { atribuciones limitadas y sólo para sancionar a sus } \\
\text { colegiados. }\end{array}$} & Mujeres & 3.1 & $-1.994 *$ & 167 & .048 \\
\hline & Hombres & 3.6 & & & \\
\hline
\end{tabular}

Nota: Se asumió la igualdad de varianzas, dada el estadístico de Levene $\mathrm{p}>0.05$

$* \mathrm{p}<.05$, dos colas.

Tabla 10. Psicólogos menores de 35 años y mayores de 36 años: Reactivo que expresa diferencias estadísticamente significativas

\begin{tabular}{|c|c|c|c|c|c|}
\hline & & Media & $T$ & $\mathrm{gl}$ & Sig \\
\hline \multirow{2}{*}{$\begin{array}{l}\text { 12. Si viera a un(a) colega enfrentando } \\
\text { una situación éticamente compleja, le } \\
\text { recomendaría que recurriera al Código de } \\
\text { Ética Profesional del Colegio de Psicólogos. }\end{array}$} & Edad $=35$ años & 3.3 & $-2.351 *$ & 167 & .020 \\
\hline & Edad $=36$ años & 3.7 & & & \\
\hline
\end{tabular}

Nota: Se asumió la igualdad de varianzas, dada la prueba de Levene $\mathrm{p}>0.05$ en los ítemes presentados.

$* \mathrm{p}<.05$, dos colas; $* * \mathrm{p}<.01$, dos colas. 
Tabla 11. Última vez que consultó el Código de Ética Profesional

\begin{tabular}{lccc}
\hline & $\begin{array}{c}\text { Colegiados } \\
(\mathrm{n}=114)\end{array}$ & $\begin{array}{c}\text { No Colegiados } \\
(\mathrm{n}=55) \# \text { Una persona no explicita si está o no afiliada } \\
\text { al Colegio de Psicólogos de Chile (A.G.).\# }\end{array}$ & $\begin{array}{c}\text { Total } \\
(\mathrm{N}=169)\end{array}$ \\
\hline Durante el último año & 50.9 & 32.7 & 45.0 \\
Hace 2 años & 7.0 & 5.3 & 7.1 \\
Hace 3 años & 2.6 & 10.9 \\
Hace 4 años & 1.8 & 3.6 & 3.6 \\
Hace 5 años & 1.8 & 7.3 & 2.7 \\
Hace más de 5 años & 7.9 & 32.7 & 7.7 \\
No responde - No recuerda & 28.1 & 29.6 \\
\hline
\end{tabular}

Tabla 12. Psicólogos/as Colegiados/as y No colegiados/as según recuerdo de Principios del Código de Ética Profesional

\begin{tabular}{lccc}
\hline & Colegiado/a & No Colegiado/a & Total \\
\hline Recuerda Principios Código de Ética Profesional & 87 & 31 & 118 \\
No recuerda o no sabe & 7 & 1 & 8 \\
No responde & 20 & 23 & 43 \\
Total Sujetos & 114 & 55 & 169 \\
\hline
\end{tabular}

Tabla 13. Psicólogos/as Colegiados/as y No colegiados/as: Reactivos que expresan diferencias estadísticamente significativas

\begin{tabular}{|c|c|c|c|c|c|}
\hline & & Media & $t$ & $\mathrm{gl}$ & Sig \\
\hline \multirow{2}{*}{$\begin{array}{l}\text { 3. Por regla general, soy contrario(a) a los códigos } \\
\text { de ética profesional. }\end{array}$} & Colegiado/a & 3.6 & $2.439 *$ & 167 & .016 \\
\hline & $\begin{array}{l}\text { No } \\
\text { Colegiado/a }\end{array}$ & 2.9 & & & \\
\hline \multirow{2}{*}{$\begin{array}{l}\text { 9. El Código de Ética Profesional del Colegio de } \\
\text { Psicólogos es una herramienta útil que orienta } \\
\text { frente a situaciones de dilema o conflicto ético. }\end{array}$} & Colegiado/a & 3.8 & $1.977 *$ & 167 & .050 \\
\hline & $\begin{array}{l}\text { No } \\
\text { Colegiado/a }\end{array}$ & 3.4 & & & \\
\hline \multirow{2}{*}{$\begin{array}{l}\text { 12. Si viera a un(a) colega enfrentando una } \\
\text { situación éticamente compleja, le recomendaría } \\
\text { que recurriera al Código de Ética Profesional del } \\
\text { Colegio de Psicólogos. }\end{array}$} & Colegiado/a & 3.7 & $3.596^{* *}$ & 167 & .000 \\
\hline & $\begin{array}{l}\text { No } \\
\text { Colegiado/a }\end{array}$ & 3.0 & & & \\
\hline
\end{tabular}

\footnotetext{
$* \mathrm{p}<.05$, dos colas; $* * \mathrm{p}<.01$ dos colas.
} 
Tabla 14. Psicólogos/as Clínicos/as y De Otras Áreas de Desempeño: Reactivos que expresan diferencias estadísticamente significativas

\begin{tabular}{|c|c|c|c|c|c|}
\hline & & Media & $t$ & $\mathrm{gl}$ & Sig \\
\hline \multirow[t]{2}{*}{$\begin{array}{l}\text { 3. Por regla general, soy contrario(a) a los códigos de ética } \\
\text { profesional. }\end{array}$} & Clínica & 3.2 & $-2.484 *$ & 148.699 & .014 \\
\hline & Otras áreas & 3.8 & & & \\
\hline \multirow[t]{2}{*}{$\begin{array}{l}\text { 4. Considero que un(a) psicólogo(a) dedicado(a) y responsable no } \\
\text { necesita recurrir al código de ética. }\end{array}$} & Clínica & 3.2 & $-2.253^{*}$ & 145.343 & .026 \\
\hline & Otras áreas & 3.7 & & & \\
\hline \multirow[t]{2}{*}{$\begin{array}{l}\text { 5. Con un buen manejo técnico basta para cubrir todos los requeri- } \\
\text { mientos éticos que entraña el ejercicio profesional de la Psicología. }\end{array}$} & Clínica & 3.2 & $-2.269 *$ & 146.425 & .025 \\
\hline & Otras áreas & 3.7 & & & \\
\hline \multirow[t]{2}{*}{$\begin{array}{l}\text { 6. La experiencia personal es lo único que me permite discernir } \\
\text { apropiadamente frente a problemas éticos en el trabajo }\end{array}$} & Clínica & 3.1 & $-2.738 * *$ & 150.625 & .007 \\
\hline & Otras áreas & 3.6 & & & \\
\hline \multirow{3}{*}{$\begin{array}{l}\text { 8. No tiene sentido poseer un Código de Ética, cuando tenemos un } \\
\text { Colegio de Psicólogos que tiene atribuciones limitadas y sólo para } \\
\text { sancionar a sus colegiados. }\end{array}$} & Clínica & & & & \\
\hline & & 3.0 & $-2.681 * *$ & 162 & .008 \\
\hline & Otras áreas & 3.6 & & & \\
\hline \multirow[t]{2}{*}{$\begin{array}{l}\text { 10. El Código de Ética Profesional del Colegio de Psicólogos aborda } \\
\text { todas las necesidades que tenemos en materias de ética profesional }\end{array}$} & Clínica & 2.8 & $2.294^{*}$ & 100.672 & .024 \\
\hline & Otras áreas & 2.4 & & & \\
\hline \multirow{2}{*}{$\begin{array}{l}\text { 12. Si viera a un(a) colega enfrentando una situación éticamente com- } \\
\text { pleja, le recomendaría que recurriera al Código de Ética Profesional } \\
\text { del Colegio de Psicólogos. }\end{array}$} & Clínica & 3.6 & $1.977 *$ & 162 & .050 \\
\hline & Otras áreas & 3.2 & & & \\
\hline
\end{tabular}

Nota: Sólo se asumió la igualdad de varianzas en los ítemes 8 y 10, dada la prueba de Levene $\mathrm{p}>0.05$

$* \mathrm{p}<.05$, dos colas; $* * \mathrm{p}<.01$, dos colas.

No obstante, se evidencia que aquellos que están colegiados/as refieren recordar los principios éticos más que los no afiliados, de manera estadísticamente significativa: $X^{2}(2, \mathrm{~N}=169)=12.17, \mathrm{p}<.01$. La Tabla 12 facilita tal comparación.

En la Tabla 13 siguiente es posible percibir que los y las psicólogas colegiadas parecieran ser más proclives a la adscripción a la norma y manifestar opiniones más favorables respecto de la utilidad del Código de Ética Profesional.

\section{Resultados según Área de desempeño}

También encontramos diferencias estadísticamente significativas por área de desempeño. Se dividió la muestra en dos grupos, uno que reúne a aquellas personas que ejercen en psicología clínica con y sin otra área adicional. En un segundo grupo se dejó a aquellos sujetos que se dedican a uno o más ámbitos, distintos de la clínica, lo que se grafica en la Tabla14.
De este modo, se observa que los/as psicólogos/as dedicados/as al ejercicio en el área clínica se muestran más propensos a adscribir a la norma comparados con aquellos/as que ejercen en otras áreas, quienes privilegiarían la responsabilidad y experiencia personal y el manejo técnico como soportes de la resolución de problemas éticos (Tabla 14).

\section{Discusión y Conclusiones}

Los resultados desplegados corroboran nuestras hipótesis en tanto se obtuvo una valoración más positiva del Código de Ética Profesional por parte de los/as psicólogos/ as clínicos/as, afiliados/as al Colegio y de mayor edad. Asimismo, la submuestra de colegiados/as recordó más Principios Éticos ante la pregunta que indagaba por ellos.

Según lo observado en los porcentajes de consulta al Código de Ética y la edad y año de titulación de los/ as psicólogos/as de la muestra, se podría pensar que accedieron al Código de Ética principalmente durante su 
formación como psicólogos/as, como parte del proceso que implica aproximarse al campo profesional y a la identidad del rol. Sin embargo, su utilización concreta -recurrir al código ante dudas éticas- se relaciona directamente con la especialidad o campo laboral en que se desempeñan los/as psicólogos/as. Se confirma en este caso, los resultados del estudio de Fassler y Rivas (2005), en la necesidad de contar con orientaciones y guías particulares que respondan a la especificidad de los diversos escenarios y roles en que actualmente se desempeñan los/as psicólogos/as en Chile.

A partir de los resultados obtenidos se puede suponer que los sujetos de la muestra anteponen otras estrategias y recursos para abordar situaciones éticamente sensibles, pudiendo manifestarse, de este modo, poco proclives a la adscripción a normas o criterios establecidos deontológicamente. Esto, especialmente en cuánto más de la mitad de los/as encuestados/as se declara contrario/a a los códigos de ética profesionales, reportando que prefieren utilizar otros recursos para la resolución de problemas éticos. Pensamos que este fenómeno es atribuible al contexto postmoderno, donde se privilegia la mirada subjetiva y la generación de códigos particulares, que se rearticulan según las circunstancias e influencias políticas e ideológicas.

Sin embargo, no contamos con información respecto de cuántos/as colegas ciertamente conocen el Código de Ética Profesional; conocen en el sentido de tener presente y manejar los principios, normas generales y normas específicas en forma tal que efectivamente puedan aplicarlas en el ejercicio profesional. Y el grado de conocimiento del código debería influir también en su valoración.

Los hallazgos expresan diferencias según el ámbito de desempeño. Quienes ejercen la psicología clínica y están colegiados/as se muestran más proclives a las normativas $\mathrm{y}$, al mismo tiempo, manifiestan una valoración favorable del Código de Ética del Colegio de Psicólogos de Chile (A. G.).

Por otra parte, al revisar los resultados según la edad de los/as encuestados/as, si bien se aprecia que existe una mayor afiliación al gremio en aquellos sujetos mayores de 36 años, no se observan mayores diferencias en lo que atañe a la adscripción a la norma y a la valoración del código, posiblemente debido a que en general se trata de una muestra joven.

En el contexto postmoderno, notamos cierta correspondencia entre los planteamientos de Sanabria (2001) -acerca del rechazo a las normas y obligaciones y la pluralidad de éticas-con los tipos de participación gremial y propensión y valoración de los códigos deontológicos que indican nuestros resultados. Por una parte, obtuvimos una muy baja tasa de respuesta y, por otra, la vinculación con el Colegio de Psicólogos opera mayoritariamente en el espacio virtual.

En tanto los códigos de ética profesional para psicó$\operatorname{logos}$ /as tienen por objetivo estimular y ayudar a los/as profesionales a actuar apropiadamente -éticamente- con sus clientes, colegas y otras personas involucradas en sus relaciones profesionales, los resultados de esta investigación evidencian la urgencia de actualizar el código chileno. Al mismo tiempo, queda pendiente la tarea de elaborar pautas y orientaciones específicas que respondan a la diversificación del quehacer profesional que nuestra disciplina ha experimentado.

Por otra parte, en un contexto de rápida diversificación y apertura de los ámbitos de acción, es posible que cada vez sea más necesario desarrollar nuevas estrategias para abordar situaciones éticamente complejas, tal como lo postula Bauman (2004). No obstante, la aspiración de resguardo y delimitación del desempeño óptimo de la profesión de los códigos deontológicos no atañe solamente a los/as profesionales, sino también a los/as futuros/as profesionales y a los/as beneficiarios/as de su quehacer; $y$, aún más, involucra a todo el entramado social (Cortina, 2003).

\section{Referencias}

Abellán, J. (2006). Sobre la vida y obra de Max Weber. En: Weber, M. La ciencia como profesión. Madrid: Espasa

Amorós, C. (1995). Feminismo, Ilustración y Posmodernidad. En: Amorós, C. Diálogos sobre Filosofía y Género. México D. F.: Universidad Nacional Autónoma de México.

Bauman, Z. (2004). Ética Posmoderna. Buenos Aires: Siglo Veintiuno Editores.

British Psychological Society (2006). Code of Ethics and Conduct. Extraído el 17 de junio de 2007, de: http://www.bps.org.uk/documentdownload-area/document-download\$.cfm?file_uuid=5084A882-1143DFD0-7E6C F1938A65C242\&ext=pdf

Canadian Psychological Association (2000). Canadian Code of Ethics for Psychologist. Ontario. Extraído el 17 de junio de 2007 de http://www. cpa.ca/cpasite/userfiles/Documents/Canadian $\% 20$ Code $\% 20$ of $\% 20$ Ethics\%20for\%20Psycho.pdf.

Canadian Psychological Association. (2007). Guidelines for Ethical Psychological Practice with Women. Extraído el 25 de Febrero de 2008, de http://www.cpa.ca/cpasite/userfiles/Documents/publications/ guidelines\%20for\%20psychological\%20practice\%20women.pdf

Colegio de Psicólogos de Chile (1975). Código de Ética Profesional. (Disponible por el Colegio de Psicólogos de Chile A. G. Ricardo Matte Pérez 492, Providencia, Santiago, Chile).

Colegio de Psicólogos de Chile (1999). Código de Ética Profesional. Presentación. Extraído el 15 marzo de 2007 de: http://www.colegiopsicologos.cl/comisiones/presentacioncodigo.pdf

Committee on Ethical Guidelines for Forensic Psychologist. (1991). Specialty Guidelines for Forensic Psychologist's. Law and Human Behavior, 16(6), p. 655-665. Extraído el 25 de Febrero de 2008, en: http://ap-ls.org/links/currentforensicguidelines.pdf

Cortina, A., Torralba, M.J. \& Zugasti, J. (1997). Ética de las profesiones. En: Arroyo, M. P. Ética y Legislación en Enfermería. Madrid: Mc-Graw Hill Interamericana.

Cortina, A. (2003). Conferencia “Ética, ciudadanía y modernidad”. Revista Cyber Humanitas $\mathrm{N}^{\circ} 27$.

Díaz, E. (1999). Posmodernidad. Buenos Aires: Biblos.

Eagleton, T. (1997). Las ilusiones del posmodernismo. Buenos Aires: Paidos.

Farrell, B., Cobbin, D. \& Farrell, H. (2002). Can codes of ethics really produce consistent behaviours? Journal of Managerial Psychology, 17 (6), 468-490.

Frassler, G. \& Rivas, M. (2005). Una Propuesta integral del Código de Psicólogos: Inclusión de lo jurídico a la legalidad de la práctica de la 
psicología. En: Escaff, E. \& Maffioletti, F. (Ed.). Psicología Jurídica. Aproximaciones desde la experiencia. Santiago: Ediciones Universidad Diego Portales.

Fernández Pérez, J. (2001). Elementos que consolidan el concepto de profesión. Notas para su reflexión. Revista electrónica de investigación educativa. 3(2). 23-39. Extraído el 15 Marzo, 2007 de http://redalyc. uaemex.mx/redalyc/pdf/155/15503202.pdf

García, C. \& Cerón, A. (2005). Entre la ética y deontología profesionales. Reflexión sobre el campo periodístico. Revista Reencuentro $\mathrm{N}^{\circ} 43$. Extraído el 16 de febrero de 2008 de http://redalyc.uaemex.mx/redalyc/ pdf/340/34004306.pdf

Gyarmati, G. (1984). Las profesiones: conocimiento y el poder. Santiago: Ediciones Universidad Católica de Chile.

Hernández, R., Férnandez, C. \& Baptista, L. (1991). Metodología de la investigación. México, D.F.: McGraw Hill.

Hirsch, A. (s/f). Ética Profesional como Proyecto de Investigación. Extraído el 27 de Octubre de 2006, de http://www.ateiamerica.com/doc/ Art\%EDculoA.\%20Hirsch.pdf

Hirsch, A. (2003). Elementos significativos de la ética profesional. Revista Reencuentro. No. 38. Extraído el 27 de octubre de 2006, de http:// redalyc.uaemex.mx/redalyc/pdf/340/34003802.pdf

Kaptein, M. \& Schwartz, M. (2008). The Effectiveness of Business Codes: A Critical Examination of Existing Studies and the Development of an Integrated Research Model. Journal of Business Ethics. 77 (2), 111-127.

Leach, M. \& Harbin, J. (1997). Psychological Ethics Codes: A Comparison of Twenty-four Countries. International Journal of Psychology, 32 (3), 181-192.

Peppas, S. (2003). Attitudes toward codes of ethics: The effects of corporate misconduct. Management Research News. 26 (6), 77-90.
Pettifor, J. (2004). Profesional Ethics Across National Boundaries. European Psychologist, 9(4), 264-272.

RAE. (2001). Diccionario de la lengua española. Vigésima Segunda Edición. Extraído el 20 de Enero 2008. Disponible en: http://buscon. rae.es/draeI/SrvltConsulta?TIPO_BUS=3\&LEMA=propender

RAE, (2007). Diccionario de la lengua española. Vigésima Tercera Edición. Extraído el 20 de Enero 2008 de http://buscon.rae.es/draeI/ SrvltConsulta?TIPO_BUS=3\&LEMA $=$ opini $\% \mathrm{C} 3 \% \mathrm{~B} 3 \mathrm{n}$

Rodríguez, M. \& Díaz, A. (2004). Códigos éticos; construcción colectiva de carácter organizacional. El caso de la Universidad Nacional de Colombia, sede Manizales. Innovar. Extraído el 10 de diciembre de 2007 de: http://www.scielo.org.co/scielo.php?script=sci_arttext\&pid=S0121$50512004000200004 \& \operatorname{lng}=$ en\&nrm $=$ iso

Sanabria, J. (2001). Ética y postmodernidad. Extraído el 16 de febrero de $2008 \mathrm{de}$ http://www.grupologosula.org/dikaiosyne/art/dik059.pdf

Schwartz, M. (2004). Effective Corporate Codes of Ethics: Perceptions of Code Users. Journal of Business Ethics. 55. 323-343.

Sinclair, C. \& Pettifor, J. (1992). Companion Manual to the Canadian Code of Ethics for Psychologist. En: Sociedad Mexicana de Psicología. Código ético del psicólogo. México D.F.: Trillas.

Sociedad Mexicana de Psicología. (2007). Código ético del psicólogo. México D.F.: Trillas.

Weber, M. (2007). La ciencia como profesión. Madrid: Espasa.

Winkler, M. I. \& Castillo, J. (en preparación). El abordaje de la ética profesional por el Colegio de Psicólogos de Chile: notas históricas. 